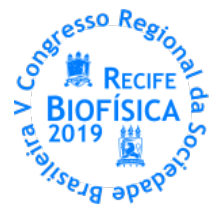

\title{
BIOCONJUGAÇÃO DE PONTOS QUÂNTICOS COM LECTINA DE BAUHINIA MONANDRA PARA APLICAÇÕES BIOLÓGICAS
}

\author{
Natália Regina Mélo Santos ${ }^{1,2}$, Weslley Felix de Oliveira ${ }^{1,2}$, Mariana Paola Cabrera ${ }^{2}$, Silvio Assis de Oliveira \\ Ferreira $^{1}$, Thiago Henrique Napoleão ${ }^{1}$, Patrícia Maria Guedes Paiva ${ }^{1}$, Márcia Vanusa da Silva ${ }^{1}$, Luana Cassandra \\ Breitenbach Barroso Coelho ${ }^{1}$, Paulo Euzébio Cabral Filho ${ }^{2}$, Adriana Fontes ${ }^{2}$, Maria Tereza dos Santos Correia ${ }^{1}$
}

${ }^{1}$ Departamento de Bioquímica, UFPE; ${ }^{2}$ Departamento de Biofísica e Radiobiologia, UFPE natalia.reginamelo@ufpe.br

\begin{abstract}
Carboidratos expressos em membranas celulares possuem importantes funções para seu metabolismo, dentre elas, destacamse, suas propriedades antigênicas, portanto, analisar sua expressão e disposição são fundamentais para entender diversos processos biológicos. É observada uma emergente associação de lectinas proteínas que se ligam reversível e especificamente a carboidratos - com nanopartículas para obtenção de gliconanossondas para estudos glicobiológicos através de técnicas baseadas em fluorescência. A lectina extraída das folhas de Bauhinia monandra (BmoLL), possuindo especificidade para o monossacarídeo galactose, dispõe de atividades biológicas já demonstradas por diversos estudos, tais como: atividades antioxidante, analgésica e anti-inflamatória, bem como ação inseticida. Os pontos quânticos (PQs) são nanocristais de semicondutores, amplamente utilizados como sondas fluorescentes, que apresentam alta resistência à fotodregadação e superfície ativa para conjugação com biomoléculas. Este trabalho teve como principal objetivo a preparação de um novo nanossistema fluorescente baseado na conjugação por adsorção da BmoLL com os PQs de telureto de cádmio (CdTe), para estudos glicobiológicos. O bioconjugado (PQsBmoLL) e os PQs sozinhos foram caracterizados por espectroscopias de absorção e emissão. A bioatividade da lectina no bioconjugado foi avaliada pelo ensaio de hemaglutinação. 0 desempenho do biconjugado foi estudado, inicialmente, com hemácias de doadores saudáveis dos tipos sanguíneos $B, A B$ e 0 , utilizadas como prova de conceito (parecer comitê de ética: 3.167 .603 do HEMOPE e 3.061.165 da UFPE). Uma suspensão de hemácias a $1 \%$ foi incubada com o bioconjugado na proporção 1:1 (hemácias/PQs-BmoLL). A citometria de fluxo foi utilizada para quantificar a porcentagem de
\end{abstract}

RESUMO marcação das hemácias pelo bioconjugado. A especificidade de ligação foi determinada incubando previamente o bioconjugado com a galactose $\left(0,2 \mathrm{~mol} \mathrm{~L}^{-1}\right)$. Como resultados, após a adição da lectina observou-se um desvio do comprimento de onda de emissão para a esquerda (blue shift), podendo ser um indício da modificação de superfície dos PQs pela interação com a BmoLL. 0 bioconjugado apresentou uma boa atividade hemaglutinante (1024 1 HUA) indicando a permanência da atividade da lectina após a bioconjugação. As análises iniciais indicaram que em doadores com hemácias do tipo $B$, que possuem na estrutura do antígeno a $D$ galactose, foi observado o maior percentual de marcação $(63,5 \%)$. Hemácias do tipo $O$ não apresentaram marcação $(0,2 \%)$, indicando uma alta especificidade do bioconjugado. Nas hemácias do tipo $A B$, que possuem simultaneamente 0 antígeno $B$ e o antígeno $A(N$ acetilgalactosamina) na membrana, mostraram marcações com menor percentual $(20,9 \%)$, possivelmente devido ao diferente padrão de exposição da galactose na superfície das hemácias $A B$. Mais estudos são necessários para confirmar e compreender essa diminuição na marcação. As análises utilizando as hemácias não só corroboraram o sucesso da conjugação da BmoLL com os PQs, como também mostraram as hemácias do tipo $B$ como um modelo eficaz para avaliar a eficiência do bioconjugado proposto. Além disso, o nanossistema fluorescente pode ser uma estratégia eficiente para a investigação e elucidação dos diferentes perfis de expressão e distribuição de carboidratos, não só na membrana eritrocitária, mas também, nos processos de infecções microbianas, patogenicidade de microrganismos e na elucidação de processos associados a células tumorais e alterações relacionadas à metástase. 